\title{
On externally complete subsets and common fixed points in partially ordered sets
}

Mohammad Z Abu-Sbeih ${ }^{1 *}$ and Mohamed A Khamsi ${ }^{1,2}$

* Correspondence:

abusbeih@kfupm.edu.sa

'Department of Mathematics \&

Statistics, King Fahd University of

Petroleum and Minerals, Dhahran

31261, Saudi Arabia

Full list of author information is

available at the end of the article

\begin{abstract}
In this study, we introduce the concept of externally complete ordered sets. We discuss the properties of such sets and characterize them in ordered trees. We also prove some common fixed point results for order preserving mappings. In particular, we introduce for the first time the concept of Banach Operator pairs in partially ordered sets and prove a common fixed point result which generalizes the classical De Marr's common fixed point theorem.

2000 MSC: primary 06F30; 46B20; 47E10.
\end{abstract}

Keywords: partially ordered sets, order preserving mappings, order trees, hyperconvex metric spaces, fixed point

\section{Introduction}

This article focuses on the externally complete structure, a new concept that was initially introduced in metric spaces as externally hyperconvex sets by Aron-szajn and Panitchpakdi in their fundamental article [1] on hyperconvexity. This idea developed from the original work of Quilliot [2] who introduced the concept of generalized metric structures to show that metric hyperconvexity is in fact similar to the complete lattice structure for ordered sets. In this fashion, Tarski's fixed point theorem [3] becomes Sine and Soardi's fixed point theorems for hyperconvex metric spaces $[4,5]$. For more on this, the reader may consult the references [6-8].

We begin by describing the relevant notation and terminology. Let $(X, \prec)$ be a partially ordered set and $M \subset X$ a non-empty subset. Recall that an upper (resp. lower) bound for $M$ is an element $p \in X$ with $m \prec p$ (resp. $p \prec m$ ) for each $m \in M$; the leastupper (resp. greatest-lower) bound of $M$ will be denoted sup $M$ (resp. $\inf M$ ). A nonempty subset $M$ of a partially ordered set $X$ will be called Dedekind complete if for any nonempty subset $A \subset M$, sup $A$ (resp $\inf A$ ) exists in $M$ provided $A$ is bounded above (resp. bounded below) in $X$. Recall that $M \subset X$ is said to be linearly ordered if for every $m_{1}, m_{2} \in M$ we have $m_{1} \prec m_{2}$ or $m_{2} \prec m_{1}$. A linearly ordered subset of $X$ is called a chain. For any $m \in X$ define

$$
(\leftarrow, m]=\{x \in X ; x \prec m\} \quad \text { and } \quad[m, \rightarrow)=\{x \in X ; m \prec x\} .
$$

Recall that a connected partially ordered set $X$ is called a tree if $X$ has a lowest point $e$, and for every $m \in X$, the subset $[e, m]$ is well ordered.

(c) 2011 Abu-Sbeih and Khamsi; licensee Springer. This is an Open Access article distributed under the terms of the Creative Commons Attribution License (http://creativecommons.org/licenses/by/2.0), which permits unrestricted use, distribution, and reproduction in any medium, provided the original work is properly cited. 
A subset $Y$ of a partially ordered set $X$ is called convex if the segment $[x, y]=\{z \in X$; $x \prec z \prec y\} \subset Y$ whenever $x, y \in Y$. A map $T: X \rightarrow X$ is order preserving (also called monotone, isotone, or increasing) if $T(x)<T(y)$ whenever $x<y$.

\section{Externally complete sets}

Inspired by the success of the concept of the externally hyperconvex subsets introduced by Aronszajn and Panitchpakdi [1], we propose a similar concept in partially ordered sets.

Definition 2.1. Let $(X, \prec)$ be a partially ordered set. A subset $M$ of $X$ is called externally complete if and only if for any family of points $\left(x_{\alpha}\right)_{\alpha \in \Gamma}$ in $X$ such that $I\left(x_{\alpha}\right) \cap I\left(x_{\beta}\right) \neq \emptyset$ for any $\alpha, \beta \in \Gamma$, and $I\left(x_{\alpha}\right) \cap M \neq \emptyset$, we have

$$
\left(\cap_{\alpha \in \Gamma} I\left(x_{\alpha}\right)\right) \cap M \neq \emptyset,
$$

Where $I(x)=(\leftarrow, x]$ or $I(x)=[x, \rightarrow)$.

The family of all nonempty externally complete subsets of $X$ will be denoted by $\mathcal{E C}(X)$.

Proposition 2.1. Let $X$ be a partially ordered set. Then, any $M \in \mathcal{E C}(X)$ is Dedekind complete and convex.

Proof. Let $A \subset M \in \mathcal{E C}(X)$ be nonempty and bounded above in $X$. The set $U(A)=\{b$ $\in X ; A \subset(\leftarrow, b]\}$ is not empty since $A$ is bounded above. It is clear that the families $(I$ $(a))_{a \in A}$, where $I(a)=[a, \rightarrow)$ and $(I(b))_{b \in U(A)}$, where $I(b)=(\leftarrow, b]$, intersect 2-by-2. Moreover, we have $I(a) \cap M \neq \emptyset$ and $I(b) \cap M \neq \emptyset$, for any $(a, b) \in A \times U(A)$. Since $M$ is in $\mathcal{E C}(X)$, we conclude that

$$
J=(\underset{a \in A}{\cap} I(a)) \cap(\underset{b \in U(A)}{\cap} I(b)) \cap M \neq \emptyset .
$$

Let $m \in J$. Then, for any $a \in A$, we have $a \prec m$. So, $m$ is an upper bound of $A$. Let $b$ be any upper bound of $A$, then $b \in U(A)$. Hence, $m \prec b$ which forces $m$ to be the least upper bound of $A$, i.e. $m=\sup A$. Similarly, one can prove that inf $A$ also exists and belongs to $M$ provided $A$ is bounded below in $X$. Next, we prove that $M$ is convex. Let $x, y \in M$. Obviously, if $x$ and $y$ are not comparable, then $[x, y]=\emptyset$ and we have nothing to prove. So, assume $x<y$. Let $a \in[x, y]$. Obviously, we have $(\leftarrow, a] \cap[a, \rightarrow)=\{a\}$. And, since $(\leftarrow, a] \cap M \neq \emptyset$ and $[a, \rightarrow) \cap M \neq \emptyset$, then

$$
(\leftarrow, a] \cap[a, \rightarrow) \cap M=\{a\} \cap M \neq \emptyset .
$$

This obviously implies that $a \in M$, i.e. $[x, y] \subset M$, which completes the proof of our proposition.

Example 2.1. Let $\mathbb{N}=\{0,1, \ldots\}$. we consider the order $0<2 \prec 4 \prec \ldots$ and $0<1<3 \prec$ ..., and no even number (different from 0 ) is comparable to any odd number. Then, ( $\mathbb{N}$, $\prec)$ is a tree. The set $M=\{0,1,2\}$ is in $\mathcal{E C}(\mathbb{N})$. Note that $M$ is convex and is not linearly ordered.

In the next result, we characterize the externally complete subsets of trees.

Theorem 2.1. Let $X$ be a tree. A subset $M$ of $X$ is externally complete if and only if $M$ is convex, Dedekind complete, and any chain $C \subset M$ has a least upper bound in $M$. 
Proof. Let $M \in \mathcal{E C}(X)$. Then, $M$ is convex and Dedekind complete. Let $C$ be a nonempty chain of $M$. Let $c_{1}, c_{2} \in C$, then we have $c_{1} \prec c_{2}$ or $c_{2} \prec c_{1}$. Hence,

$$
\left[c_{1}, \rightarrow\right) \cap\left[c_{2}, \rightarrow\right) \cap M \neq \emptyset .
$$

Since $M \in \mathcal{E C}(X)$

$$
J=(\underset{c \in C}{\cap}[c, \rightarrow)) \cap M \neq \emptyset .
$$

Obviously, any $c \in J$ is an upper bound of $C$. Since $M$ is Dedekind complete, sup $C$ exists in $M$. Assume conversely that $M$ is a convex, and Dedekind complete subset of $X$ such that any chain in $M$ has an upper bound in $M$. Let $x, y \in X$ such that there exist $m_{1}, m_{2} \in M$ with $x \prec m_{1}$ and $m_{2} \prec y$. Define $P(x)=\inf \{m \in M ; x<m\}$, and $P(y)$ $=\sup \{m \in M ; m \prec y\}$. Both $P(x)$ and $P(y)$ exist and belong to $M$ since $M$ is Dedekind complete. Let $\left(x_{i}\right)_{i \in I}$ in $X$ be such that for any $i \in I$ there exists $m_{i} \in M$ such that $x_{i} \prec$ $m_{i}$. Also, we have $\left[x_{i}, \rightarrow\right) \cap\left[x_{j}, \rightarrow\right) \neq \emptyset$, for any $i, j \in I$. This condition forces the set $\left\{x_{i} ; i \in I\right\}$ to be linearly ordered since $X$ is a tree. Consider the subset $M_{I}=\left\{P\left(x_{i}\right) ; i \in\right.$ $I$ ) of $M$. It is easy to check that $M_{I}$ is linearly ordered. Since any linearly ordered subset of $M$ is bounded above, there exists $m \in M$ such that $P\left(x_{i}\right) \prec m$ for any $i \in I$. Since $x_{i}$ $\prec P\left(x_{i}\right)$ then

$$
m \in\left(\bigcap_{i \in I}\left[x_{i}, \rightarrow\right)\right) \cap M \neq \emptyset .
$$

Next, let $\left(y_{j}\right)_{j \in J}$ in $X$ such that for any $j \in J$ there exists $m_{j} \in M$ such that $m_{j} \prec y_{j}$. Consider the subset $M_{J}=\left\{P\left(y_{j}\right) ; j \in \mathcal{J}\right\}$ of $M$. Since $X$ is a tree, the set $M_{J}$ is bounded below, so $m_{0}=\inf M_{J}$ exists in $M$. It is obvious that $m_{0} \prec P\left(y_{j}\right) \prec y_{j}$ for any $j \in J$. This implies

$$
m_{0} \in\left(\bigcap_{j \in J}\left(\leftarrow, y_{j}\right]\right) \cap M=\emptyset .
$$

Finally, assume that we have $\left(x_{i}\right)_{i \in I}$ and $\left(y_{j}\right)_{j \in J}$ in $X$ such that the subsets $\left(\left[x_{i}, \rightarrow\right)\right)_{i \in I}$, and $\left(\left(\leftarrow, y_{j}\right]\right)_{j \in J}$ intersect 2 -by-2 and $\left[x_{i}, \rightarrow\right) \cap M \neq \emptyset$ and $\left(\leftarrow, y_{j}\right] \cap M \neq \emptyset$ for any $(i, j)$ $\in I \times J$. As before, set

$$
m_{I}=\sup \left\{P\left(x_{i}\right) ; i \in I\right\} \quad \text { and } \quad m_{J}=\inf \left\{P\left(y_{j}\right) ; j \in J\right\} .
$$

For any $(i, j) \in I \times J$,we have $P\left(x_{i}\right) \prec P\left(y_{j}\right)$, which implies $m_{I} \prec m_{J}$. Obviously we have

$$
\left[m_{I}, m_{J}\right] \subset\left(\bigcap_{i \in I}\left[x_{i}, \rightarrow\right)\right) \cap\left(\bigcap_{i \in I}\left(\leftarrow, y_{j}\right]\right) \cap M \neq \emptyset .
$$

Hence, $M$ is in $\mathcal{E C}(X)$.

The above proof suggests that externally complete subsets are proximinal. In fact in [1], the authors introduced externally hyperconvex subsets as an example of proximinal sets other than the admissible subsets, i.e. intersection of balls. Before we state a similar result, we need the following definitions.

Definition 2.2. Let $X$ be a partially ordered set. Let $M$ be a nonempty subset of $X$. Define the lower and upper cones by

$$
\mathcal{C}_{l}(M)=\{x \in X ; \text { there exists } m \in M \text { such that } x \prec m\}
$$


and

$$
\mathcal{C}_{u}(M)=\{x \in X \text {; there exists } m \in M \text { such that } m \prec x\} .
$$

The cone generated by $M$ will be defined by $\mathcal{C}(M)=\mathcal{C}_{l}(M) \cup \mathcal{C}_{u}(M)$.

Theorem 2.2. Let $X$ be a partially ordered set and $M$ a nonempty externally complete subset of $X$. Then, there exists an order preserving retract $P: \mathcal{C}(M) \rightarrow$ Msuch that

(1) for any $x \in \mathcal{C}_{l}(M)$ we have $x<P(x)$, and

(2) for any $x \in \mathcal{C}_{u}(M)$ we have $P(x) \prec x$.

Proof. First set $P(m)=m$ for any $m \in M$. Next, let $x \in \mathcal{C}_{l}(M)$. We have

$$
x \in[x, \rightarrow) \cap(\underset{x<m}{\cap}(\leftarrow, m]),
$$

where $m \in M$. Using the external completeness of $M$, we get

$$
M \cap[x, \rightarrow) \cap(\underset{x<m}{\cap}(\leftarrow, m]) \neq \emptyset .
$$

It is easy to check that this intersection is reduced to one point. Set

$$
M \cap[x, \rightarrow)(\underset{x<m}{\cap}(\leftarrow, m])=\{P(x)\} .
$$

Similarly, let $x \in \mathcal{C}_{u}(M)$. We have

$$
x \in(\leftarrow, x] \cap(\underset{m<x}{\cap}[m, \rightarrow)),
$$

where $m \in M$. Using the external completeness of $M$, we get

$$
M \cap(\leftarrow, x] \cap(\underset{m<x}{\cap}[m, \rightarrow)) \neq \emptyset .
$$

It is easy to check that this intersection is reduced to one point. Set

$$
M \cap(\leftarrow, x] \cap(\underset{m<x}{\cap}[m, \rightarrow))=\{P(x)\} .
$$

In particular, this prove (1) and (2). In order to finish the proof of the theorem, let us show that $P$ is order preserving. Indeed, let $x, y \in \mathcal{C}(M)$ with $x \prec y$. If $y \in \mathcal{C}_{l}(M)$, then $x \in \mathcal{C}_{l}(M)$. Since $y \prec P(y)$ then $x \prec P(y)$ which implies $P(x) \prec P(y)$. Similarly, if $x \in \mathcal{C}_{u}(M)$, then $y \in \mathcal{C}_{u}(M)$ and again it is easy to show $P(x) \prec P(y)$. Assume $x \in \mathcal{C}_{l}(M)$ and $y \in \mathcal{C}_{u}(M)$. Since

$$
x \in[x, \rightarrow) \cap(\underset{x<m}{\cap}(\leftarrow, m]) \cap(\leftarrow, y]
$$

and $M$ is externally complete, we have

$$
M \cap[x, \rightarrow) \cap(\underset{x<m}{\cap}(\leftarrow, m]) \cap(\leftarrow, y] \neq \emptyset,
$$


where $m \in M$. But $[x, \rightarrow) \cap(\underset{x<m}{\cap}(\leftarrow, m])=\{P(x)\}$, this forces $P(x) \in(\leftarrow, y]$. By definition of $P(y)$, we get $P(x) \prec P(y)$. In fact, we proved that $x \prec P(x) \prec P(y) \prec y$. This completes the proof of the theorem.

We have the following result.

Theorem 2.3. Let $X$ be a partially ordered set and $M$ a nonempty externally complete subset of $X$. Assume that $X$ has a supremum or an infimum, then there exists an order preserving retract $P: X \rightarrow M$ which extends the retract of $\mathcal{C}(M)$ into $M$.

Proof. Let $P: \mathcal{C}(M) \rightarrow M$ be the retract defined in Theorem 2.2. Assume first that $X$ has a supremum $e$. Then, $X=\mathcal{C}_{l}(\mathcal{C}(M))$. Indeed, for any $x \in X$, we have $x<e$ and $e \in \mathcal{C}_{u}(M)$. Because $x \in \bigcap_{x<z}(\leftarrow, z]$, where $z \in \mathcal{C}_{u}(M)$, and $M$ is externally complete, we get $M \cap\left(\bigcap_{x \prec z}(\leftarrow, z]\right) \neq \emptyset$, where $z \in \mathcal{C}_{u}(M)$. Hence, there exists $m \in M$ such that $m \prec$ $z$, for any $z \in \mathcal{C}_{u}(M)$ such that $x<z$. Using the properties of $P$, we get $m \prec P(z)$, for any $z \in \mathcal{C}_{u}(M)$ such that $x \prec z$. Since $M$ is Dedekind complete, $\inf \left\{P(z) ; z \in \mathcal{C}_{u}(M)\right.$ such that $x \prec z\}$ exists. Set

$$
\tilde{P}(x)=\inf \left\{P(z) ; z \in \mathcal{C}_{u}(M) \text { such that } x \prec z\right\} .
$$

First note that if $x \in \mathcal{C}(M)$, then for any $z \in \mathcal{C}_{u}(M)$ such that $x \prec z$ we have $P(x) \prec P$ $(z)$. This will imply $P(x) \prec \tilde{P}(x)$. If $x \in \mathcal{C}_{u}(M)$, then by definition of $\tilde{P}$, we have $\tilde{P}(x) \prec P(x)$. Hence, $P(x) \prec \tilde{P}(x)$. If $x \in \mathcal{C}_{l}(M)$, then by definition of $\tilde{P}$, we have $\tilde{P}(x) \prec P(P(x))$ since $x \prec P(x)$. Hence, $\tilde{P}(x) \prec P(x)$ which implies again $P(x) \prec \tilde{P}(x)$. So, $\tilde{P}$ extends $P$. Let us show that $\tilde{P}$ is order preserving. Indeed, let $x, y \in X$ such that $x \prec$ $y$. Since

$$
\left\{P(z) ; z \in \mathcal{C}_{u}(M) \text { such that } y \prec z\right\} \subset\left\{P(z) ; z \in \mathcal{C}_{u}(M) \text { such that } x \prec z\right\}
$$

we have

$$
\inf \left\{P(z) ; z \in \mathcal{C}_{u}(M) \text { such that } x \prec z\right\} \prec \inf \left\{P(z) ; z \in \mathcal{C}_{u}(M) \text { such that } y \prec z\right\},
$$

or $\tilde{P}(x) \prec \tilde{P}(y)$. In order to finish the proof of Theorem 2.3, consider the case when $X$ has an infimum, say $e$. Then, $X=\mathcal{C}_{l}(\mathcal{C}(M))$. As for the previous case, define

$$
\tilde{P}(x)=\sup \left\{P(z) ; z \in \mathcal{C}_{l}(M) \text { such that } z \prec x\right\} .
$$

It is easy to show that $\tilde{P}(x)$ exists. In a similar proof, one can show that $\tilde{P}$ extends $P$ and is order preserving.

Since a tree has an infimum, we get the following result.

Corollary 2.1. Let $X$ be a tree and $M$ a nonempty externally complete subset of $X$. Then, there exists an order preserving retract $P: X \rightarrow M$.

A similar result for externally hyperconvex subsets of metric trees maybe found in [9].

\section{Common fixed point}

In this section, we investigate the existence of a common fixed point of a commuting family of order preserving mappings defined on a complete lattice. Here the proof follows the ideas of Baillon [10] developed in hyperconvex metric spaces. It is amazing that these ideas extend nicely to the case of partially ordered sets. The ideas in 
question are not the conclusions which maybe known but the proofs as developed in the metric setting. Maybe one of the most beautiful results known in the hyperconvex metric spaces is the intersection property discovered by Baillon [10]. The boundedness assumption in Baillon's result is equivalent to the complete lattice structure in our setting. Indeed, any nonempty subset of a complete lattice has an infimum and a supremum. We have the following result in partially ordered sets.

Theorem 3.1. Let $X$ be a partially ordered set. Let $\left(\mathrm{X}_{\beta}\right)_{\beta \in \Gamma}$ be a decreasing family of nonempty complete lattice subsets of $X$, where $\Gamma$ is a directed index set. Then, $\cap_{\beta \in \Gamma} X_{\beta}$ is not empty and is a complete lattice.

Proof. Consider the family

$$
\mathcal{F}=\left\{\prod_{\beta \in \Gamma} A_{\beta} ; A_{\beta} \text { is a nonempty interval in } X_{\beta} \text { and }\left(A_{\beta}\right) \text { is decreasing }\right\} .
$$

$\mathcal{F}$ is not empty since $\prod_{\beta \in \Gamma} X_{\beta} \in \mathcal{F}$. In a complete lattice, any decreasing family of nonempty intervals has a nonempty intersection and it is an interval. Therefore, $\mathcal{F}$ satisfies the assumptions of Zorn's lemma. Hence, for every $D \in \mathcal{F}$, there exists a minimal element $A \in \mathcal{F}$ such that $A \subset D$. We claim that if $\prod_{\beta \in \Gamma} A_{\beta}$ is minimal, then each $A_{\beta}$ is a singleton. Indeed, let us fix $\beta_{0} \in \Gamma$. We know that $\mathrm{A}_{\beta 0}=\left[m_{\beta 0}, M_{\beta 0}\right]$. Consider the new family

$$
B_{\beta}\left\{\begin{array}{cc}
A_{\beta} & \text { if } \beta_{0} \prec \beta \text { or } \beta \text { not comparable to } \beta_{0}, \\
\left\{x \in X_{\beta} ; m_{\beta_{0}} \prec x \prec M_{\beta_{0}}\right\} & \text { if } \beta \prec \beta_{0}
\end{array}\right.
$$

Our assumptions on $\left(X_{\beta}\right)$ and $\left(A_{\beta}\right)$ imply that $\left(B_{\beta}\right) \in \mathcal{F}$. Moreover, we have $B_{\beta} \subset A_{\beta}$ for any $\beta \in \Gamma$. Since $\prod_{\beta \in \Gamma} A_{\beta}$ is minimal, we get $B_{\beta}=A_{\beta}$ for any $\beta \in \Gamma$. In particular, we have

$$
A_{\beta}=\left\{x \in X_{\beta} ; m_{\beta_{0}} \prec x \prec M_{\beta_{0}}\right\}
$$

for $\beta \prec \beta_{0}$. If $A_{\beta}=\left[m_{\beta}, M_{\beta}\right]$, then we must have $m_{\beta}=m_{\beta 0}$ and $M_{\beta}=M_{\beta 0}$. Therefore, we proved the existence of $m, M \in X$ such that $A_{\beta}=\left\{x \in X_{\beta} ; m \prec x<M\right\}$, for any $\beta \in$ $\Gamma$. It is easy from here to show that in fact we have $m=M$ by the minimality of $\Pi_{\beta \in \Gamma}$ $A_{\beta}$, which proves our claim. Clearly, we have $m \in A_{\beta}$ for any $\beta \in \Gamma$ which implies $K=$ $\cap_{\beta \in \Gamma} X_{\beta}$ is not empty. Next, we will prove that $K$ is a complete lattice. Let $A \subset K$ be nonempty. We will only prove that $\sup A$ exists in $K$. The proof for the existence of the infimum follows identically. For any $\beta \in \Gamma$, we have $A \subset X_{\beta}$. Since $X_{\beta}$ is a complete lattice, then $m_{\beta}=\sup A$ exists in $X_{\beta}$. The interval $\left[m_{\beta}, \rightarrow\right)$ is a complete lattice. Clearly, the family $\left(\left[m_{\beta}, \rightarrow\right)\right)_{\beta \in \Gamma}$ is decreasing. From the above result, we know that $n_{\beta \in \Gamma}\left[m_{\beta}, \rightarrow\right)$ is not empty. Therefore, there exists $m \in K$ such that $a<m$ for any $a \in$ $A$. Set $B=\{m \in K ; a \prec m$ for any $a \in A\}$. For any $\beta \in \Gamma$, define $M_{\beta}=\inf B$ in $X_{\beta}$. Set

$$
X_{\beta}^{*}=\bigcap_{a \in A, b \in B}[a, b] \bigcap X_{\beta}=\left[m_{\beta}, M_{\beta}\right] \cap X_{\beta} .
$$

Then, $X_{\beta}^{*}$ is a nonempty complete sublattice of $X_{\beta}$. It is easy to see that the family $\left(X_{\beta}^{*}\right)$ is decreasing. Hence, $\cap_{\beta \in \Gamma} X_{\beta}^{*}$ is not empty Obviously, we have 


$$
\bigcap_{\beta \in \Gamma} X_{\beta}^{*}=\{\sup A\}
$$

in $n_{\beta \in \Gamma} X_{\beta}$. The proof of Theorem 3.1 is therefore complete.

As a consequence of this theorem, we obtain the following common fixed point result.

Theorem 3.2. Let $X$ be a complete lattice. Then, any commuting family of order preserving mappings $\left(T_{i}\right)_{i \in I}, T_{i}: X \rightarrow X$, has a common fixed point. Moreover, if we denote by Fix $\left(\left(T_{i}\right)\right)$ the set of the common fixed points, then Fix $\left(\left(T_{i}\right)\right)$ is a complete sublattice of $X$.

Proof. First note that Tarski fixed point theorem [3] implies that any finite commuting family of order preserving mappings $T_{1}, T_{2}, . ., T_{n}, T_{i}: X \rightarrow X$, has a common fixed point. Moreover, if we denote by $\operatorname{Fix}\left(\left(T_{i}\right)\right)$ the set of the common fixed points, i.e. Fix $\left(\left(T_{i}\right)\right)=\left\{x \in M ; T_{i}(x)=x i=1, . ., n\right\}$, is a complete sublattice of $X$. Let $\Gamma=\{\beta ; \beta$ is a finite nonempty subset of $I\}$. Clearly, $\Gamma$ is downward directed (where the order on $\Gamma$ is the set inclusion). For any $\beta \in \Gamma$, the set $F_{\beta}$ of common fixed point set of the mappings $T_{i}, i \in \beta$, is a nonempty complete sublattice of $X$. Clearly, the family $\left(F_{\beta}\right)_{\beta \in \Gamma}$ is decreasing. Theorem 3.1 implies that $n_{\beta \in \Gamma} F_{\beta}$ is nonempty and is a complete sublattice of $X$. The proof of Theorem 3.2 is therefore complete.

The commutativity assumption maybe relaxed using a new concept discovered in [11] (see also [12-15]. Of course, this new concept was initially defined in the metric setting, therefore we need first to extend it to the case of partially ordered sets.

Definition 3.1. Let $X$ be a partially ordered set. The ordered pair $(S, T)$ of two selfmaps of the set $X$ is called a Banach operator pair, if the set Fix $(T)$ is S-invariant, namely $S(F i x(T)) \subseteq F i x(T)$.

We have the following result whose proof is easy.

Theorem 3.3. Let $X$ be a complete lattice. Let $T: X \rightarrow X$ be an order preserving mapping. Let $S: X \rightarrow X$ be an order preserving mapping such that $(S, T)$ is a Banach operator pair. Then, $\operatorname{Fix}(S, T)=\operatorname{Fix}(T) \cap \operatorname{Fix}(S)$ is a nonempty complete lattice.

In order to extend this conclusion to a family of mappings, we will need the following definition.

Definition 3.2. Let $T$ and $S$ be two self-maps of a partially ordered set $X$. The pair $(S, T)$ is called symmetric Banach operator pair if both $(S, T)$ and $(T, S)$ are Banach operator pairs, i.e., $T(F i x(S)) \subseteq F i x(S)$ and $S(F i x(T)) \subseteq F i x(T)$.

We have the following result which can be seen as an analogue to De Marr's result [16] without compactness assumption of the domain.

Theorem 3.4. Let $X$ be a partially ordered set. Let $\mathcal{T}$ be a family of order preserving mappings defined on $X$. Assume any two mappings from $\mathcal{T}$ form a symmetric Banach operator pair. Then, the family $\mathcal{T}$ has a common fixed point provided one map from Thas a fixed point set which is a complete lattice. Moreover, the common fixed point set Fix $(\mathcal{T})$ is a complete lattice.

Proof. Let $T_{0} \in \mathcal{T}$ be the map for which $\operatorname{Fix}\left(T_{0}\right)=X_{0}$ is a nonempty complete lattice. Since any two mappings from $\mathcal{T}$ form a symmetric Banach operator pair, then for any $T \in \mathcal{T}$, we have $T\left(X_{0}\right) \subset X_{0}$. Since $X_{0}$ is a complete lattice, $T$ has a fixed point in $X_{0}$. The fixed point set of $T$ in $X_{0}$ is $\operatorname{Fix}(T) \cap \operatorname{Fix}\left(T_{0}\right)$ and is a complete sublattice of $X_{0}$. Let $S \in \mathcal{T}$. Then, $S\left(\operatorname{Fix}(T) \cap \operatorname{Fix}\left(T_{0}\right)\right) \subset \operatorname{Fix}(T) \cap \operatorname{Fix}\left(T_{0}\right)$. Since $\operatorname{Fix}(T) \cap \operatorname{Fix}\left(T_{0}\right)$ is a 
complete lattice, then $S$ has a fixed point in $\operatorname{Fix}(T) \cap \operatorname{Fix}\left(T_{0}\right)$. The fixed point set of $S$ in $\operatorname{Fix}(T) \cap \operatorname{Fix}\left(T_{0}\right)$ is $\operatorname{Fix}(S) \cap \operatorname{Fix}(T) \cap \operatorname{Fix}\left(T_{0}\right)$ which is a complete sublattice of $X_{0}$. By induction, one will prove that any finite subfamily $T_{1}, \ldots, T_{n}$ of $\mathcal{T}$ has a nonempty common fixed point set $\operatorname{Fix}\left(T_{1}\right) \cap \cdots \cap \operatorname{Fix}\left(T_{n}\right) \cap X_{0}$ which is a complete sublattice of $X_{0}$. Theorem 3.1 will then imply that $\bigcap_{T \in \mathcal{T}} \operatorname{Fix}(T) \cap X_{0}$ is not empty and is a complete lattice. Since

$$
\operatorname{Fix}(\mathcal{T}) \cap X_{0}=\operatorname{Fix}(\mathcal{T}),
$$

we conclude that $\operatorname{Fix}(\mathcal{T})$ is a nonempty complete lattice.

\section{Acknowledgements}

The authors were grateful to King Fahd University of Petroleum \& Minerals for supporting research project IN 101008.

\section{Author details}

'Department of Mathematics \& Statistics, King Fahd University of Petroleum and Minerals, Dhahran 31261, Saudi Arabia ${ }^{2}$ Department of Mathematical Sciences, The University of Texas at El Paso, El Paso, TX 79968, USA

\section{Authors' contributions}

All authors participated in the design of this work and performed equally. All authors read and approved the final manuscript.

\section{Competing interests}

The authors declare that they have no competing interests.

Received: 21 August 2011 Accepted: 6 December 2011 Published: 6 December 2011

\section{References}

1. Aronszajn, N, Panitchpakdi, P: Extensions of uniformly continuous transformations and hyperconvex metric spaces. Pacific J Math. 6, 405-439 (1956)

2. Quilliot, A: Homomorphismes, points fixes, rtractions et jeux de poursuite dans les graphes, les ensembles ordonns et les espaces mtriques, Thèse de Doctorat d'Etat. Paris (1983)

3. Tarski, A: A lattice theoretical fixpoint theorem and its applications. Pacific J Math. 5, 285-309 (1955)

4. Sine, R: On nonlinear contraction semigroups in sup norm spaces. Nonlinear Anal Theory Methods Appl. 3, 885-890 (1979). doi:10.1016/0362-546X(79)90055-5

5. Soardi, P: Existence of fixed points of nonexpansive mappings in certain Banach lattices. Proc Amer Math Soc. 73 25-29 (1979). doi:10.1090/S0002-9939-1979-0512051-6

6. Jawhari, E, Misane, D, Pouzet, M: Retracts: graphs and ordered sets from the metric point of view. Contemp Math. 57, 175-226 (1986)

7. Khamsi, MA, Misane, D: Fixed point theorems in logic programming. Ann Math Artif Intell. 21, 231-243 (1997). doi:10.1023/A:1018969519807

8. Khamsi, MA, Kirk, WA, Martinez Yanez, C: Fixed point and selection theorems in hyper-convex spaces. Proc Amer Math Soc. 128, 3275-3283 (2000). doi:10.1090/S0002-9939-00-05777-4

9. Aksoy, AG, Khamsi, MA: A selection theorem in metric trees. Proc Amer Math Soc. 134, 2957-2966 (2006). doi:10.1090/ S0002-9939-06-08555-8

10. Baillon, JB: Nonexpansive mappings and hyperconvex spaces. Contemp Math. 72, 11-19 (1988)

11. Chen, J, Li, Z: Common fixed points for Banach operator pairs in best approximation. J Math Anal Appl. 336, 1466-1475 (2007). doi:10.1016/j.jmaa.2007.01.064

12. Espinola, R, Hussain, N: Common fixed points for multimaps in metric spaces. Fixed Point Theory Appl 14 (2010). 2010, Article ID 204981

13. Hussain, $\mathrm{N}$ : Common fixed points in best approximation for Banach operator pairs with Ciric type I-contractions. J Math Anal Appl. 338, 1351-1362 (2008). doi:10.1016/j.jmaa.2007.06.008

14. Pathak, HK, Hussain, N: Common fixed points for Banach operator pairs with applications. Nonlinear Anal. 69, 2788-2802 (2008). doi:10.1016/j.na.2007.08.051

15. Hussain, N, Khamsi, MA, Latif, A: Banach operator pairs and common fixed points in hyperconvex metric spaces Nonlinear Anal TMA. 74(17), 5956-5961 (2011). doi:10.1016/j.na.2011.05.072

16. De Marr, R: Common fixed points for commuting contraction mappings. Pacific J Math. 13, 1139-1141 (1963)

doi:10.1186/1687-1812-2011-97

Cite this article as: Abu-Sbeih and Khamsi: On externally complete subsets and common fixed points in partially ordered sets. Fixed Point Theory and Applications 2011 2011:97. 